\title{
Another Piece of Evidence for Early Administration of Biologics in Children with Crohn's Disease Who Start as an Inflammatory Phenotype
}

\author{
Hyuk Yoon
}

Department of Internal Medicine, Seoul National University Bundang Hospital, Seongnam, Korea

\author{
Corresponding Author \\ Hyuk Yoon \\ ORCID https://orcid.org/0000-0002-2657-0349 \\ E-mail bodnsoul@hanmail.net
}

\begin{abstract}
See "Risk Factors for Disease Behavior Evolution and Efficacy of Biologics in Reducing Progression in Pediatric Patients with Nonstricturing, Nonpenetrating Crohn's Disease at Diagnosis: A Single-Center Experience in Korea" by Hyun Jin Kim, et al. on page 851, Vol. 15, No. 6, 2021
\end{abstract}

A quarter of inflammatory bowel disease cases occur in children, and Crohn's disease is much more common than ulcerative colitis in children. Therefore, Crohn's disease is significant in the field of pediatric gastroenterology. The inflammatory phenotype is relatively common than the stricturing or penetrating phenotype in children compared to adults. Therefore, it can be said that there is a higher chance of preventing disease progression in pediatric Crohn's disease. On the other hand, caution should be exercised because pediatric Crohn's disease takes a more aggressive disease course than adults. ${ }^{1}$ Regarding this issue, the recent Selecting Therapeutic Targets in Inflammatory Bowel Disease (STRIDE) II recommends taking the same treat-to-target approach for pediatric Crohn's disease as adults. ${ }^{2}$ However, the evidence for this suggestion is weaker than for adults. A Western pediatric study reported that early use of biologics delayed the progression to stricturing and penetrating disease. ${ }^{3}$ However, studies about Asian children are rare, and there are debates about the efficacy of biologics. A recent review paper stated that early biologic therapy could be suggested in Asian patients with Crohn's disease who have high-risk factors for the poor outcome; there was no mention of pediatric Crohn's disease. ${ }^{4}$

In this issue of Gut and Liver, Kim et al. ${ }^{5}$ reported risk factors for disease behavior evolution in pediatric patients with nonstricturing, nonpenetrating Crohn's disease at diagnosis. Among 402 pediatric patients who initially had inflammatory phenotype, the disease progressed into stricturing or penetrating phenotype in 75 patients (18.7\%; follow-up period, median 6.1 years). The cumulative probabilities of disease behavior evolution were $34.3 \%$ at 10 years. Patients whose disease progressed had an increased risk of intestinal resection (hazard ratio [HR], $3.61 ; 95 \%$ confidence interval [CI], 2.25 to 6.03 ; $\mathrm{p}<0.001$ ). First-degree family history of inflammatory bowel disease (HR, 2.38; 95\% CI, 1.07 to 5.28; $\mathrm{p}=0.032$ ), isolated ileal involvement at diagnosis ( $\mathrm{HR}, 7.55$; 95\% CI, 1.04 to 15.57 ; $\mathrm{p}=0.045)$, and positive anti-Saccharomyces cerevisiae antibody titers (HR, 2.10; 95\% CI, 1.03 to 4.25; p=0.040) were associated with disease behavior evolution. Early treatment with tumor necrosis factor $\alpha$ (TNF- $\alpha$ ) inhibitors significantly reduced disease progression (HR, 0.46; 95\% CI, 0.79 to $3.39 ; \mathrm{p}=0.042$ ).

This study suggests that early aggressive therapy should be considered in pediatric Crohn's disease patients who have inflammatory phenotype and risk factors of disease evolution to improve long-term outcomes. Interestingly, isolated ileal involvement at diagnosis was associated with a high risk of behavior progression. It has been argued that Crohn's disease should be classified into ileum dominant Crohn's disease and isolated colonic Crohn's disease since they have different disease nature. ${ }^{6}$ In addition, there is a suggestion that even small bowel Crohn's disease should be further subdivided according to the dominant lesion. ${ }^{7} \mathrm{Pe}$ diatric gastroenterologists might be more familiar with this issue since they have been already subdividing children who have a location of L4 into L4a and L $4 b{ }^{8}$ Therefore, it 
is expected that additional studies comparing prognosis according to the dominant lesion at diagnosis in small bowel Crohn's disease will be conducted in children first.

In summary, this study is an excellent one that adds another piece of evidence to the claim that early administration of biologics should be considered in children with Crohn's disease who start as an inflammatory phenotype but have risks factors for disease progression. TNF- $\alpha$ inhibitors were the only biologics used in this study. There is a recent movement to expand the use of various biologics in children as in adults. Therefore, researches on whether early administration of other biologics such as integrin inhibitors or IL-12/23 inhibitors is also effective in preventing disease progression in pediatric Crohn's disease will be needed soon.

\section{CONFLICTS OF INTEREST}

No potential conflict of interest relevant to this article was reported.

\section{ORCID}

Hyuk Yoon https://orcid.org/0000-0002-2657-0349

\section{REFERENCES}

1. Moon JS. Clinical aspects and treatments for pediatric in- flammatory bowel disease. Intest Res 2019;17:17-23.

2. Turner D, Ricciuto A, Lewis A, et al. STRIDE-II: an update on the Selecting Therapeutic Targets in Inflammatory Bowel Disease (STRIDE) Initiative of the International Organization for the Study of IBD (IOIBD): determining therapeutic goals for treat-to-target strategies in IBD. Gastroenterology 2021;160:1570-1583.

3. Kerur B, Machan JT, Shapiro JM, et al. Biologics delay progression of Crohn's disease, but not early surgery, in children. Clin Gastroenterol Hepatol 2018;16:1467-1473.

4. Ooi CJ, Hilmi I, Banerjee R, et al. Best practices on immunomodulators and biologic agents for ulcerative colitis and Crohn's disease in Asia. Intest Res 2019;17:285-310.

5. Kim HJ, Oh SH, Lee SH, et al. Risk factors for disease behavior evolution and efficacy of biologics in reducing progression in pediatric patients with nonstricturing, nonpenetrating Crohn's disease at diagnosis: a single-center experience in Korea. Gut Liver 2021;15:851-857.

6. Dulai PS, Singh S, Vande Casteele N, et al. Should we divide Crohn's disease into ileum-dominant and isolated colonic diseases? Clin Gastroenterol Hepatol 2019;17:2634-2643.

7. Watanabe K. Clinical management for small bowel of Crohn's disease in the treat-to-target era: now is the time to optimize treatment based on the dominant lesion. Intest Res 2020;18:347-354.

8. Levine A, Griffiths A, Markowitz J, et al. Pediatric modification of the Montreal classification for inflammatory bowel disease: the Paris classification. Inflamm Bowel Dis 2011;17:1314-1321. 